\title{
System Development of I-Card for Students using Barcode
}

\author{
Sagarjit Roy \\ Faculty Of Management Studies \\ ICFAI UNIVERSITY, Tripura
}

\author{
Partha Sarathi De \\ Faculty Of Science \& Technology \\ ICFAI UNIVERSITY, Tripura
}

\author{
Debalina Saha \\ Faculty Of Management \\ ICFAI UNIVERSITY, Tripura
}

\begin{abstract}
:
The system ICGS (I-card generating system) is based on computerization of Student identity card generation system. To develop this project front-end tool as VISUAL STUDIO 2005 \& back-end tool as relational database ${ }^{1}$ has been used. Using this software user can easily print student identity information along with a unique barcode in a uniform format. Even a user can reprint an identity card instantly if needed from previously stored records. The system enables a good user-interface for the user to maintain the records. It will also provide a permanent database and this will help to store the student identity information in an error free and non redundant way along with better option for maintaining the records.
\end{abstract}

\section{Keywords :}

Barcode, Database, ID-Card, Redundant.

\section{INTRODUCTION:}

\subsection{Motivation:}

The present 21 st century is a digital era. Now-a-days the development in various aspects of computer technology has reached beyond our imagination \& expectations. Every new day, new software is launched in the market. Software has an attractive user interface \& easy maintenance facility which helps us in our working field. In certain University of Tripura students don't have any printed I-card and the card it has not carry so much of information. This fact inspired us to develop software which will aid in computerization of identity card generation system.

\subsection{Advantages to Using Barcodes}

The "height" of a Barcode contains no information, but the higher each bar is made, the easier it may be to read when presented to a reader. About 0.4 inch $(1 \mathrm{~cm})$ centered on the make off with reader sensor, should usually be sufficient.
Problems to consider include probable security breaches due to the ease of copying the code, and the potential for phy sical damage to the Barcode after repeated swipes. The major lead of a $2 \mathrm{D}$ Barcode is its data encoding competence, with up to 500 bytes per square inch being practical. Some of this data will normally be used for error correction encoding which also makes the 2D Barcode remarkably tolerant of holes, cuts, and dirt marks. With around 500 bytes of data available, a 2D Barcode can be used to store biometric data such as a fingerprint, or even a compressed version of the holder's portrait. Other promise includes detailed personalized data such as name, address, department, employee number, access authorizations, training status, and expiring date. Since swiping is not required, and 2D Barcodes are very tolerant of artifacts, physical wear should not be a problem, but a potential downside is the higher cost of scanners1. Visual Studio 2005 released on February 2002. In the past, Visual Basic has been criticized \& maligned as a "toy" language as it did not provide all of the Features of more sophisticated languages such as $\mathrm{C}++$ and JAVA although Visual Basic 1.0 had already then changed the face of windows Programming by removing the complex burden of writing code for the User Interface (UI). VB 1.0 freed the programmers to draw their own UI. Microsoft has removed all the restrictions and made VB.NET as a powerful development tool ${ }^{2}$.

\section{RELATED WORK:}

\subsection{Proposed Methodology:}

A software life cycle is a series of identifiable stages that a software product undergoes during its lifetime. A software life cycle model is a descriptive and diagrammatic representation of the software life cycle. A life cycle model maps the different activities performed on software product from its inception to retirement into a set of life cycle phases.

\footnotetext{
1 "Relational Database", http: www.techfaq.com/relational-database.html, [cited at $12^{\text {th }}$ feb2011] ${ }^{2}$ http://www.barcodefor.us/,[accessed at 12feb 2011]
} 


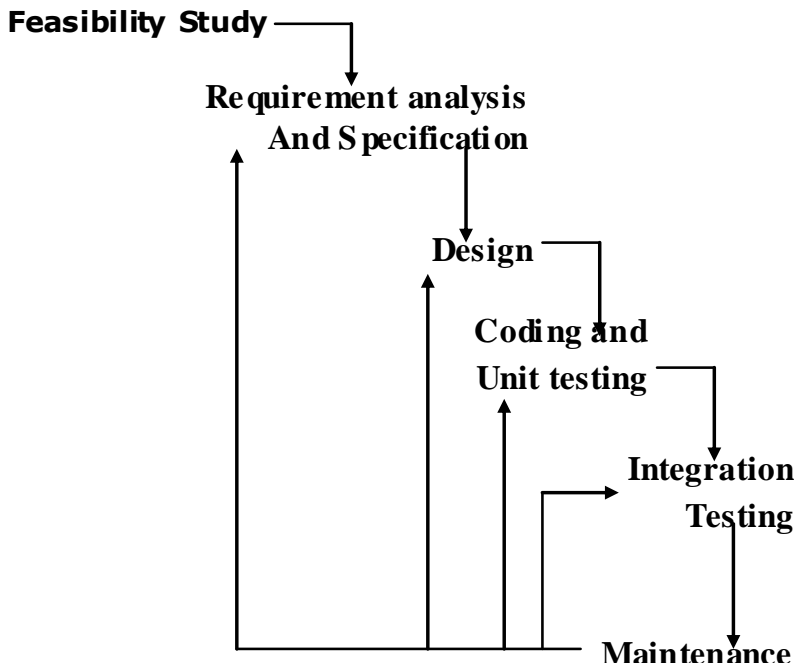

Figure1 (Iterative Waterfall Model) ${ }^{3}$

\subsection{Phase Analysis:}

During the feasibility phase, first of all the task was to find out what are the requirements for the project, and come up with a solution that to generate an identity card of a student it require the particular details of the student such as name, ID number, blood group, batch, program, an passport-size photograph etc. Next to generate the barcode the font named "IDAutomationHC39M" 3 is required, and the most important and the primary requirement of my project is a reporting software such as Crystal Report in Visual Studio 2005.Thus during the Requirement analysis \& specification phase we collected all the data that would be the input to the system i.e. student details, their photographs etc. and for the back-end i.e. the database, gathered information about the formats to store the information, and then documented all the requirements after analyzing them in a uniform way. At the designing phase the database which contains designing of tables and setting up of the table fields, data types, required fields, unique keys, primary keys etc. Later on the task designed the interface that contains designing of different forms, choosing color schemes, choosing fonts for the text to be displayed on the screens etc. The information that is documented in the previous phase has been used in these forms and for the database.

During the coding and unit testing phase the system design has divided into modules, and start writing codes for those modules, after writing codes for each module, and tested each of them to check whether it is working correctly or not.

\subsection{E-R Diagram Analysis:}

The Entity-Relationship (E-R) model is a high-level conceptual data model developed by Chen to facilitate database design. A conceptual data model is a set of concepts that describe the structure of a database and the associated retrieval and update transactions on the database. The main purpose of developing a high level data model is to support a user's perception of the data and to conceal the more technical aspects with database design. The basic model of E-R model includes entity types, relationship types and attributes.

\footnotetext{
${ }^{3}$ Rajiv Mall, “Soft ware Engineering”, PHI publication.PP23
}

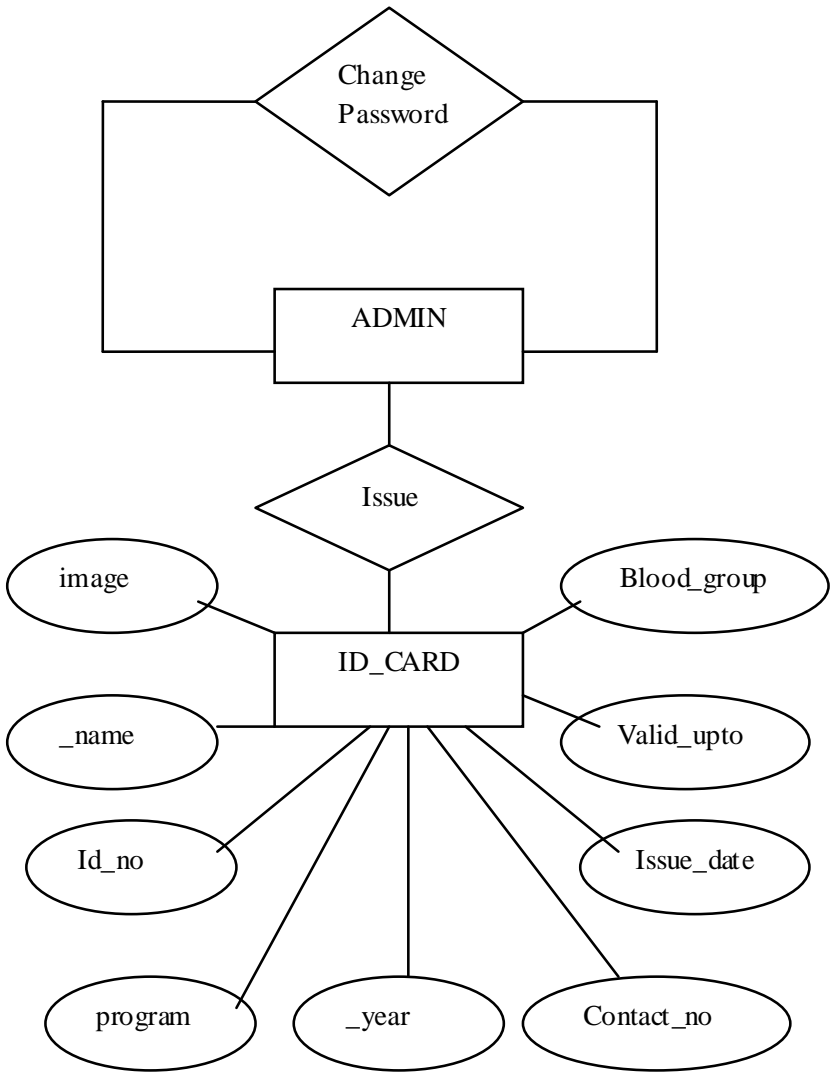

Figure 2 (E-R MODEL)

Here the two entities are ADMIN and ID_CARD and the relationship between the two of them is to issue an identity card for particular student. The ADMIN entity has two attributes namely - username and password through username and password the user or administrator can log in to the system and can issue an identity card for student. As indicated in the ER diagram, the attribute password is underlined to show that it's the primary attribute for the entity ADMIN in the sense, that the password is unique for the particular administrator. In this context, to issue an id-card for student, particular details of the student is necessary such as name, image, id number, blood group, batch, program etc. thus the entity ID_CARD has the following attributes namely - image, _name, id_no, program, year, contact_no, bloodgroup, date_of_issue (the date on which the id-card is issued) \& validupto (the date up to which the id-card is valid). The attribute id_no is primary key in the ID_CARD entity because for each student the id_no is unique i.e. for ICFAI University the enrollment number given to each of the student is unique and valid throughout the duration of the course.

\subsection{Data Flow Diagrams (DFDs) Analysis:}

A Data Flow Diagram is a hierarchical graphical model of a system that shows the different processing activities or functions that the system performs and the data interchange among those functions. The Data Flow Diagram (DFD) also known as bubble chart is a simple graphical formalism that can be used to represent a system in terms of the input data to the system, various processing carried out on those data and the output data generated by the system. The main reason why the DFD technique is so popular is probably because of the fact that DFD is a very simple formalism-it is simple to understand and 
use. A DFD model uses very limited number primitive sy mbols to represent the functions performed by a system and the data flow among these functions. In order to $\log$ in to the sy stem few functions need to be carried out, these are: reads the username and password and validate the username and password and if the username \& password is valid then log into the system. In order to save data in the database few functions need to be carried out, these are: reads the information that are filled, validate the information, if the information is valid then save them in the database. In order to search a record of a particular student from the database few functions need to be carried out, these are: reads the id number that has been given as a input by the user, validate the id number, if the id number is valid and exist in the database then return the details.

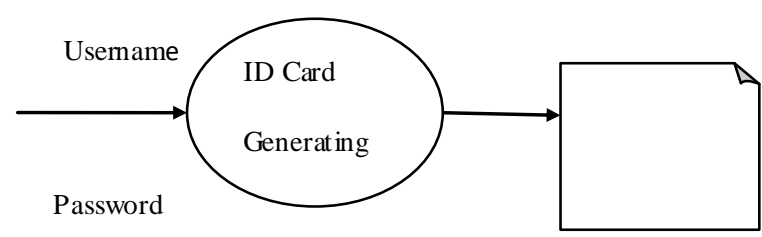

Figure 3 (0 Level DFD FOR I-CARD)

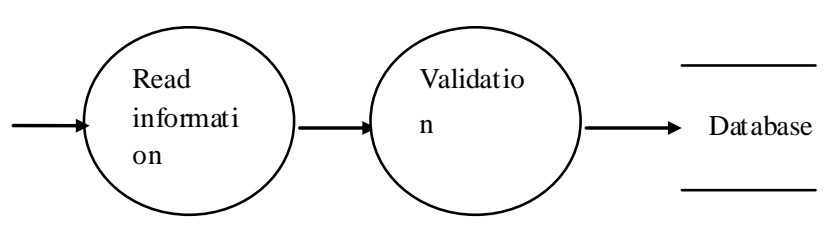

Figure 4(1 level DFD FORSAVE DATA)

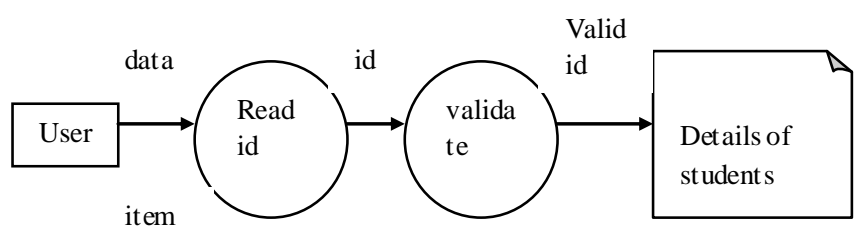

Figure 5(1 level DFD FORS EARCH DATA)

\subsection{Flow Chart:}

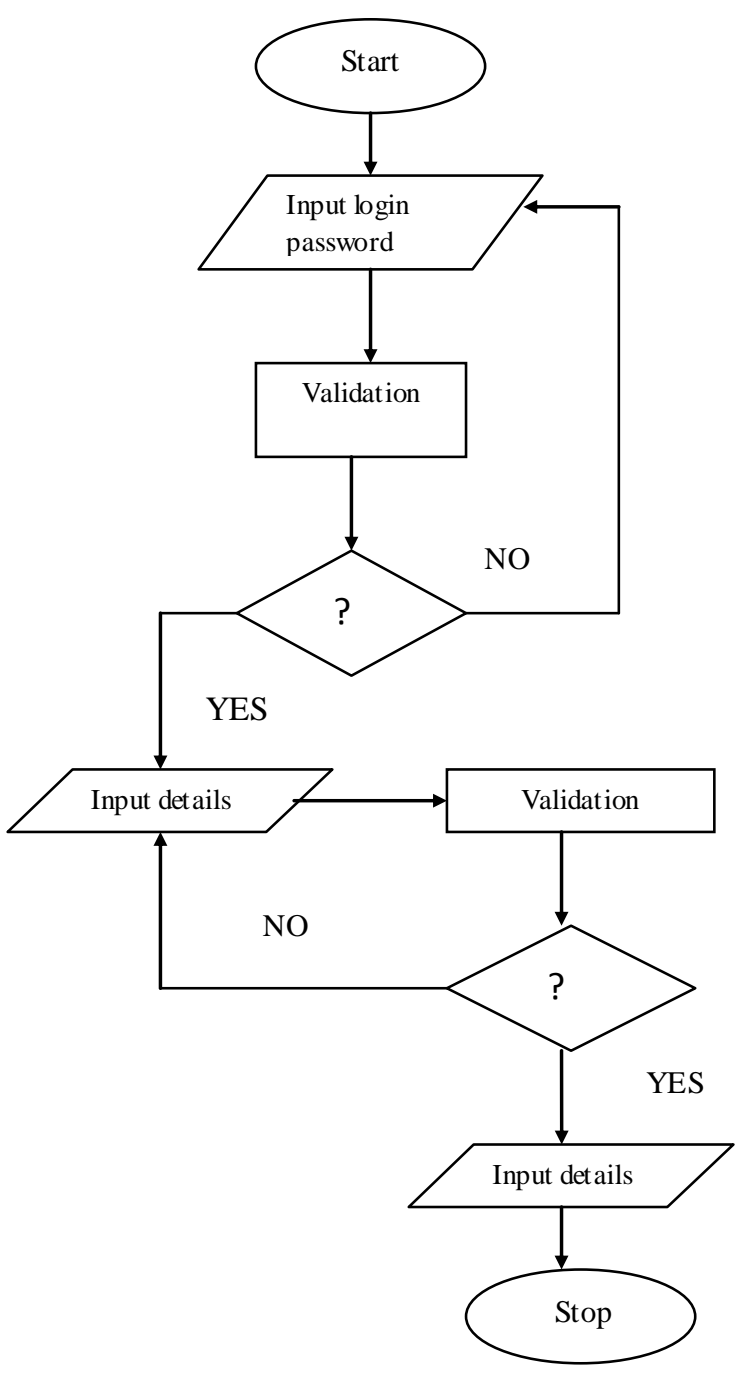

Figure 6 (Flow chart of workflow)

\subsection{User Interface Analysis:}

A fundamental reality of application development is that the user interface is the system to the users. What users want is for developers to build applications that meet their needs and that are easy to use. User interface design is important for several reasons. First of all the more intuitive the user interface the easier it is to use, and the easier it is to use and the less expensive to use it. The better the user interface the easier it is to train people to use it, reducing training costs. The better the user interface the less help people will need to use it, reducing the support costs. The better the user interface the more the users will like to use it, increasing their satisfaction with the work that has been done. The most important thing that I believe to make the user interface consistent and easy to use, thus the first thing I tried to do is to Put all the buttons in consistent places on all the forms and use the same font for all the texts that are to be displayed on the screen and used the white color scheme throughout, so to make it soothing to the user eyes and brain to enable them to build an accurate mental model of the way the system works. The next thing was to Word the messages and labels effectively, as we know the text that displays on the screens is a primary source of information for the users. If the text is worded poorly, then the interface will 
be perceived poorly by the users. Using full words and sentences, as opposed to abbreviations and codes, makes the text easier to understand.

\section{CONCLUSION:}

In the conclusion we can say that it will be very effective and user friendly to use. Even it will be very low cost to maintain the system even to print the card too. The system will provide an error free user interface so that all the manual error has been taken care .However, ICGS is enriched with barcode generating system to let the students have access to entire system in an easier way i.e. taking book from library, returning the book etc.

\section{FUTURE WORK:}

In future, the system can easily print the Identity card format in a standard way, using crystal report; the system will work very smooth with amount of data. Even using

webcam, the system can be able to take pictures which can be used in generating identity card.

\section{REFERENCES:}

[1] Rajiv Mall, "Software Engineering", PHI publication.

[2] V.Rajaraman, "Analysis and Design of information systems, second edition".PP 24-30

[3 ] V.Rajaraman, "Self- Study Guidance to Analysis and Design of Information System".pp 33
[4] Eliason, Alan L. , "Systems Development Analysis and Implementation', Litle Brown \& Company.PP

[5] Fairley, Richard, "Software Engineering Concept", McGraw-Hill.pp 23

[6] “ID card-printiing,"http://www.plasticcardid.com/school-idcard-printers.htm<accesed at $12 \mathrm{feb}, 2011>$

[7]"Smart card printing", http://www.codeproject.com/KB/smart/smartcardapi.asp $\mathrm{x}<$ accessed at $21^{\text {st }}$ Feb 2011>

[8]"Barode print" < http://www.neody namic.com/DesktopDefault.asp x $><$ acces sed at $21^{\text {st }}$ feb 2011>

[9]" 10 Principles of the UI Design Masters" http://net.tutsplus.com/articles, <accessed at $1^{\text {st }}$ March 2011>

[10]" A Summary of Principles for User-Interface Design.”,Talin,http://www.sylvantech.com/ talin/projects/ ui_design.htm<accessed at $1^{\text {st }}$ march>

[11] Alexon Leon, DataBase Management System,Leon Vikas,2005,pp 125-131

[12] Professional VB.NET 2003, Bill Evjan,Billy Hollis ,Rama Ramachandra,Bill Sheldon, Wrox Publication.

[13] Beginner's Visual Basic 2005 ,Thearon Willis,Bryan Newsome, Wrox Publication.,pp 123 